\title{
Simulating stochastic dynamics using large time steps
}

\author{
O. Corradini* \\ Dipartimento di Fisica, Università degli Studi di Bologna and INFN Sezione di Bologna, Via Irnerio 46, Bologna I-40126, Italy \\ P. Faccioli ${ }^{\dagger}$ \\ Dipartimento di Fisica, Università degli Studi di Trento and INFN, Via Sommarive 14, Povo, Trento I-38050, Italy \\ and Institut de Physique Théorique (IPhT), Centre d'Etudes de Saclay, CEA, F-91191 Gif-sur-Yvette, France \\ H. Orland \\ Institut de Physique Théorique (IPhT), Centre d'Etudes de Saclay, CEA, F-91191 Gif-sur-Yvette, France
}

(Received 22 July 2009; published 9 December 2009)

\begin{abstract}
We present an approach to investigate the long-time stochastic dynamics of multidimensional classical systems, in contact with a heat bath. When the potential energy landscape is rugged, the kinetics displays a decoupling of short- and long-time scales and both molecular dynamics or Monte Carlo (MC) simulations are generally inefficient. Using a field theoretic approach, we perform analytically the average over the short-time stochastic fluctuations. This way, we obtain an effective theory, which generates the same long-time dynamics of the original theory, but has a lower time-resolution power. Such an approach is used to develop an improved version of the MC algorithm, which is particularly suitable to investigate the dynamics of rare conformational transitions. In the specific case of molecular systems at room temperature, we show that elementary integration time steps used to simulate the effective theory can be chosen a factor $\sim 100$ larger than those used in the original theory. Our results are illustrated and tested on a simple system, characterized by a rugged energy landscape.
\end{abstract}

DOI: 10.1103/PhysRevE.80.061112

PACS number(s): 05.40.-a, 87.15.ak, 87.15.Cc, 87.15.H-

\section{INTRODUCTION}

The investigation of a vast class of physical phenomena requires the understanding of the long-time dynamics of classical systems, in contact with a heat-bath. Examples include critical dynamics, molecular aggregation, and protein folding, to name a few.

The most natural strategy to describe these processes is to integrate numerically the equations of motion, i.e., to perform molecular dynamics (MD) simulations. Unfortunately, when the number of degrees of freedom is very large, or in the presence of large free energy barriers, MD approaches become extremely costly [1], or even impracticable. The problem arises because the time scale associated with the system's local conformational changes can be many orders of magnitude smaller that the time scales of the dynamics one is interesting in studying. As a result, most of the computational time is invested in simulating uninteresting thermal oscillations.

This situation is exemplified in Fig. 1, where we show the stochastic motion of a point particle, interacting with a twodimensional external potential. The solid line was obtained by means of a MD simulation and illustrates how, at shorttime scales, the dynamics of this system is dominated by fast modes associated to thermal diffusion. However, when the evolution of the system is described using much lower timeresolution power, the effect of such short-time thermal fluc-

\footnotetext{
*olindo.corradini@bo.infn.it

†accioli@science.unitn.it

thenri.orland@cea.fr
}

tuations tends to average out and to become unimportant. This is evident from the comparison between the solid line and the dashed line, which was obtained by averaging over blocks of consecutive frames in the original MD trajectory. At long times, the dynamics of system is mostly sensitive to the structure of the external energy landscape, which was chosen to be spherically symmetric.

Clearly, an important question to ask is whether it is possible to develop theoretical/computational frameworks which yield directly the correct long dynamics, but avoid investing computational time in simulating the short-time thermal os-

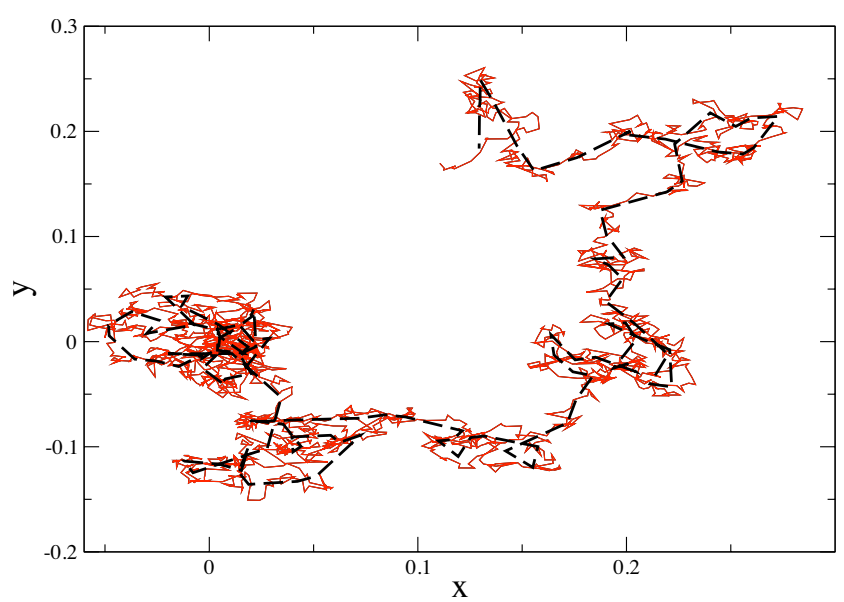

FIG. 1. (Color online) Langevin dynamics of a point particle in a two-dimensional external potential. The solid line denotes the result of an MD simulation. The dashed line is the result of averaging over blocks of consecutive frames of the MD trajectory. Such an average smooths out the trajectory. 
cillations. Mori-Zwanzig's projection techniques represent a powerful method to remove rapidly varying degrees of freedom, in large dimensional systems [2]. This method has been also applied to average out fast degrees of freedom in small systems exhibiting chaotic behavior [3]. A potential limitation of such approaches is that they imply the a priori identification of the set of slowly varying degrees of freedom.

A promising alternative to projection techniques is represented by the approaches based on Markov state models [5-7]. A potential difficulty of such approaches resides in correct identification of the metastable states. In addition, for each different system, one needs to perform a large set of independent MD simulations in order to accurate calculation of the rate coefficients.

In this work, we present an alternative approach to simulate the dynamics over long times. We develop a rigorous effective theory which (i) yields by construction the correct long dynamics and (ii) does not require to identify metastable states, nor to evaluate the transition matrix by MD. We rely on the path integral representation of the stochastic dynamics generated by the overdamped Langevin equation. A pioneering analysis of the result of integrating out fast modes from such a stochastic path integral was performed in [4], in the context of the study of phase synchronization, and with an emphasis on the geometrical implications. In this work, we are interested in constructing an efficient algorithm to perform numerical simulation long-time dynamics. To this goal, we use a field theory approach, based on renormalization group (RG) ideas and on the notion of effective field theory [8]. Such a powerful tools have been already successfully applied to describe the low-energy dynamics of a vast variety of quantum and statistical systems characterized by a separation of scales-see, e.g., $[9,10]$. To the best of our knowledge, this method has never been applied to develop an effective theory to efficiently simulate the long-time stochastic dynamics of a system in contact with a heat bath.

The main idea of our approach is to exploit the decoupling of time scales in the system in order to define a perturbative series, in which the expansion parameter is the ratio of short-over large time scales. In such a perturbative framework, the average over the short-time fluctuations can be computed analytically, to any desired level of accuracy. The average over the fast thermal oscillations gives rise to new terms in the stochastic path integral, which represent corrections both to the interaction and to the diffusion coefficient. Such new terms implicitly take into account of the dynamics of the fast degrees of freedom, which have been integrated out from the system.

Once a finite number of such effective terms corresponding to a given accuracy have been calculated analytically, it is possible to simulate the dynamics of the system using much larger time steps. By construction, in the regime of decoupling of fast and slow modes, one is guaranteed that the effective long-time theory generates the same probability distributions of the underlying, more fundamental stochastic theory. It is important to emphasize the fact that the present approach is not equivalent to simply including higher-order corrections in the Trotter expansion [11]. Indeed, the assumption of decoupling of time scales leads to further simplifications with respect to such an approach.
The paper is organized as follows. In Sec. II, we review the path integral formulation of the Langevin dynamics and we outline the formal connection between stochastic dynamics and evolution of a quantum particle in imaginary time. Such a connection is used in Sec. III to identify and isolate the dynamics of the fast degrees of freedom. In Secs. IV and $\mathrm{V}$ we present our perturbative scheme which allows to integrate out the fast modes and derive the effective interactions and diffusion coefficients. In Sec. VI we discuss how the effective theory for the dynamics at long-time scales can be simulated using the diffusion MC algorithm, which is briefly reviewed in Appendix B. Section VII is devoted to simple examples, which illustrate how this method works in practice. In Sec. VIII we discuss the applicability of the present approach to simulate the Langevin dynamics of molecular systems. Results and conclusions are summarized in Sec. IX.

\section{LANGEVIN DYNAMICS}

We consider a system defined by a stochastic $d$-dimensional variable $x$ obeying the Langevin equation,

$$
m \ddot{x}=-\nabla U(x)-\gamma \dot{x}+\xi(t),
$$

where $U(x)$ is a potential energy function, $m$ is the mass, $\gamma$ is the friction coefficient and $\xi(t)$ is a $\delta$-correlated Gaussian noise. In many molecular systems of interest, the acceleration term $m \ddot{x}$ is damped at time scales of the order $10^{-13} \mathrm{~s}$, which much smaller than the time scale associated to local conformational changes. If such a term is dropped one obtains the so-called overdamped or velocity Langevin equation,

$$
\dot{x}=-\frac{1}{\gamma} \nabla U(x)+\eta(t),
$$

where $\eta(t)$ is a rescaled delta-correlated Gaussian noise, satisfying the fluctuation-dissipation relationship,

$$
\left\langle\eta\left(t^{\prime}\right) \eta(t)\right\rangle=2 d \frac{1}{\beta \gamma} \delta\left(t-t^{\prime}\right) .
$$

The overdamped Langevin equation defines a Markovian process. The probability distribution $P(x, t)$ generated by such a stochastic differential equation obeys the FokkerPlanck equation,

$$
\frac{\partial}{\partial t} P(x, t)=\frac{1}{\beta \gamma} \nabla[\nabla P(x, t)+\beta \nabla U(x) P(x, t)] .
$$

By performing the substitution

$$
P(x, t)=\exp \left[-\frac{\beta}{2} U(x)\right] \psi(x, t)
$$

the Fokker-Planck Eq. (4) can be recast in the form of a Schrödinger equation in imaginary time,

$$
-\frac{\partial}{\partial t} \psi(x, t)=\hat{H}_{e f f} \psi(x, t),
$$

where the effective "quantum Hamiltonian” operator reads 


$$
\hat{H}_{e f f}=-\frac{1}{\beta \gamma} \nabla^{2}+\beta V_{e f f}(x),
$$

and $V_{\text {eff }}(x)$ is called the effective potential and reads

$$
V_{\text {eff }}(x)=\frac{1}{4 \gamma}\left\{[\nabla U(x)]^{2}-\frac{2}{\beta} \nabla^{2} U(x)\right\} .
$$

Hence, the problem of studying the diffusion of a classical particle can be mapped into the problem of determining the quantum-mechanical propagation in imaginary time of a virtual system, defined by the effective quantum Hamiltonian (7), interacting with the effective potential $V_{\text {eff }}(x)$.

Let $G\left(x_{f}, t_{f} \mid x_{i}\right)$ be the Green's function of the FokkerPlanck operator, subject to initial condition $x(0)=x_{i}$, i.e.,

$$
\begin{aligned}
& \frac{\partial}{\partial t} G\left(x_{f}, t \mid x_{i}\right)-\frac{1}{\beta \gamma} \nabla\left[\nabla G\left(x_{f}, t \mid x_{i}\right)+\beta \nabla U G\left(x_{f}, t \mid x_{i}\right)\right] \\
& \quad=\delta(t) \delta\left(x_{f}-x_{i}\right) .
\end{aligned}
$$

The interpretation of such a Green's function is the probability for the system to be in $x_{f}$ at $t$, conditioned to start from $x_{i}$ at the initial time. Formally, such a conditional probability can be related to the "quantum" propagator of the effective Hamiltonian (7),

$$
\begin{gathered}
G\left(x, t \mid x_{i}\right)=\exp \left[-\frac{\beta}{2}\left(U(x)-U\left(x_{i}\right)\right)\right] K\left(x, t \mid x_{i}\right), \\
K\left(x, t \mid x_{i}\right)=\left\langle x\left|e^{-t H_{e f f}}\right| x_{i}\right\rangle .
\end{gathered}
$$

Hence, it is immediate to derive a path integral representation of the Green's function $G\left(x, t \mid x_{i}\right)$,

$$
G\left(x, t \mid x_{i}\right)=e^{-\beta / 2\left(U(x)-U\left(x_{i}\right)\right)} \int_{x\left(t_{i}\right)=x_{i}}^{x(t)=x} \mathcal{D} x e^{-\beta S_{e f f}[x]},
$$

where $S_{\text {eff }}[x]$ is the effective "action,"

$$
S_{e f f}[x]=\int_{0}^{t} d \tau\left(\frac{\gamma}{4} \dot{x}^{2}+V_{e f f}(x)\right) .
$$

The prefactor $e^{-\beta / 2\left(U(x)-U\left(x_{i}\right)\right)}$ in Eq. (11) can be transformed away, noticing that $\frac{d U(x)}{d \tau}=\dot{x} U^{\prime}(x)$. One than obtains a path integral in which the statistical weight contains the OnsagerMachlup functional

$$
\begin{aligned}
G\left(x, t \mid x_{i}\right)= & \int_{x(0)=x_{i}}^{x(t)=x} \mathcal{D} x \exp -\beta \int_{0}^{t} d \tau\left[\frac{\gamma}{4} \dot{x}^{2}+\frac{1}{2} \dot{x} U^{\prime}(x)\right. \\
& \left.+V_{\text {eff }}(x)\right]
\end{aligned}
$$

We note that as long as Eqs. (12), (13), and (8) are written in the continuum limit, they are independent on the prescription adopted to define the time derivative in the original Langevin Eq. (2) - see, e.g., the discussion in [16]. On the other hand, the dependence on the choice of the stochastic calculus appears after the time variable is discretized. In the present work we consider the discretized definition of the effective action functional,

$$
\begin{aligned}
S_{e f f}[x]= & \Delta \tau \sum_{n=0}^{N-1} \frac{\gamma}{4}\left(\frac{x_{n+1}-x_{n}}{\Delta \tau}\right)^{2}+\frac{1}{4 \gamma}\left\{\left[\nabla U\left(x_{n}\right)\right]^{2}\right. \\
& \left.-\frac{2}{\beta} \nabla^{2} U\left(x_{n}\right)\right\},
\end{aligned}
$$

which corresponds to the Stratonovich midpoint prescription.

Equation (12) provides an expression for the conditional probability in terms of the microscopic stochastic dynamics governing the system. It represents the starting point of the dominant reaction pathway approach [12-15], which deals with the problem of finding the most probable transition pathways between the given configurations $x_{i}$ and $x_{f}$, which are visited at the initial and final time $x(t)=x_{f}, x(0)=x_{i}$, respectively.

On the other hand, in this work we are interested in the corresponding initial value problem, i.e., we want to develop an effective theory which yields directly the long-time evolution of the probability density $P(x, t)$, solution of Eq. (4), starting from a given initial probability density $P(x, t=0)$ $=\rho_{0}(x)$. The probability density $P(x, t)$, the Green's function $G\left(x_{f}, t \mid x_{i}, t_{i}\right)$ and the initial distribution $\rho_{0}(x)$ are related by the equation,

$$
P(x, t)=\int d y G(x, t \mid y) \rho_{0}(y) .
$$

Hence, for positive time intervals, the conditional probability $G\left(x, t \mid x_{i}\right)$ can be considered as the propagator associated to the stochastic Fokker-Planck Eq. (4).

\section{SEPARATION OF FAST AND SLOW MODES}

Without loss of generality, let us focus on the stochastic path integral [Eq. (12)], with periodic boundary conditions,

$$
\begin{aligned}
Z(t) & \equiv \int d x G(x, t \mid x, 0) \\
& =\oint \mathcal{D} x \exp \left[-\beta \int_{0}^{t} d \tau\left(\frac{\gamma}{4} \dot{x}^{2}+V_{e f f}(x)\right)\right] .
\end{aligned}
$$

We observe that the inverse temperature $\frac{1}{\beta}$ plays the role of $\hbar$, in the analogy with the quantum-mechanical formalism. Hence, the loop expansion of the path integral [Eq. (17)] generates an expansion in powers of $\frac{1}{\beta}$.

Let us introduce the Fourier conjugate,

$$
\begin{gathered}
\widetilde{x}\left(\omega_{n}\right)=\frac{1}{t} \int_{0}^{t} d \tau \exp \left[-i \omega_{n} t\right] x(\tau), \\
x(\tau)=x(\tau+t)=\sum_{n} \widetilde{x}\left(\omega_{n}\right) \exp \left[i \omega_{n} t\right],
\end{gathered}
$$

where $\omega_{n}$ are the Matsubara frequencies,

$$
\omega_{n}=\frac{2 \pi}{t} n, \quad n=0, \pm 1, \pm 2, \ldots .
$$

In numerical simulations, the integration of the overdamped Langevin equation is performed by choosing a finite 
elementary time step $\Delta t$. In frequency space, this implies the existence of an ultraviolet cutoff $\Omega$, which is inversely proportional to $\Delta t$,

$$
\Omega \sim \frac{2 \pi}{\Delta t}
$$

Such a relationship becomes a strict equality in the case of periodic boundary conditions, as in Eq. (17). In general, when the boundary conditions are not periodic, it represents just an order-of-magnitude estimate of the largest Fourier frequencies, which are associated to a given choice of the integration time step $\Delta t$.

Let us now introduce a parameter $0<b<1$ and split the frequency interval $(0, \Omega)$ as $(0, b \Omega) \cup(b \Omega, \Omega)$. Then the Fourier decomposition of a path contributing to Eq. (17) can be split as

$$
x(t)=x_{>}(t)+x_{<}(t),
$$

where $x_{<}(t)$ and $x_{>}(t)$ will be referred to as the slow and fast modes, respectively,

$$
\begin{gathered}
x_{<}(t)=\sum_{\left|\omega_{n}\right| \leq b \Omega} \tilde{x}\left(\omega_{n}\right) e^{i \omega_{n} t}, \\
x_{>}(t)=\sum_{b \Omega \leq\left|\omega_{n}\right| \leq \Omega} \tilde{x}\left(\omega_{n}\right) e^{i \omega_{n} t} .
\end{gathered}
$$

The main purpose of this work is to develop a perturbation series to systematically integrate out from the path integral the modes with frequencies $\omega_{n}>b \Omega$. To this end, we begin by rewriting the "kinetic" term which appears in the effective action (13) of the path integral [Eq. (12)] as a sum of the kinetic energy of slow and fast modes,

$$
\begin{aligned}
\frac{\gamma}{4} \int_{0}^{t} d \tau \dot{x}^{2} & =\frac{t \gamma}{4} \sum_{\left|\omega_{n}\right| \leq \Omega} \omega_{n}^{2} \widetilde{x}\left(\omega_{n}\right) \tilde{x}\left(-\omega_{n}\right) \\
& =\frac{\gamma}{4} \int_{0}^{t} d \tau \dot{x}_{<}^{2}(\tau)+\frac{\gamma t}{4} \sum_{\left|\omega_{n}\right| \in S_{b}} \omega_{n}^{2} \tilde{x}\left(\omega_{n}\right) \widetilde{x}\left(-\omega_{n}\right),
\end{aligned}
$$

where $S_{b}$ denotes the shell of hard modes $S_{b}=(b \Omega, \Omega)$.

Let us now consider the potential term and expand around the slow modes $x_{<}(t)$,

$$
\begin{aligned}
V_{e f f}[x(\tau)]= & V_{e f f}\left[x_{<}(\tau)\right]+\frac{\partial V_{e f f}\left[x_{<}(\tau)\right]}{\partial x^{i}} x_{>}^{i}(\tau) \\
& +\frac{1}{2} \frac{\partial^{2} V_{e f f}\left[x_{<}(\tau)\right]}{\partial x^{i} x^{j}} x_{>}^{i}(\tau) x_{>}^{j}(\tau)+\mathcal{O}\left(x_{>}^{3}\right)
\end{aligned}
$$

The complete path integral [Eq. (17)] can be split in the following way:

$$
\begin{aligned}
Z(t) & =\oint \mathcal{D} x_{<} \oint \mathcal{D} x_{>} e^{-\beta S_{e f f}\left[x_{<}(t)+x_{>}(t)\right]} \\
& \equiv \oint \mathcal{D} x_{<} e^{-\beta S_{e f f}\left[x_{<}(t)\right]} e^{-\beta S_{>}\left[x_{<}(\tau)\right]} .
\end{aligned}
$$

In this expression, the action functional $S_{\text {eff }}$ is evaluated on the slow modes only and depends on the original effective potential $V_{\text {eff }}$ (which we also shall refer to as the "tree-level" effective potential). $S_{>}\left[x_{<}(\tau)\right]$ is a correction term action which accounts for the dynamics of the fast modes which are integrated out,

$$
\begin{aligned}
e^{-\beta S_{>}[x<(\tau)]} \equiv & \oint \mathcal{D} x_{>} \exp \left[-\frac{\beta \gamma t}{4} \sum_{\left|\omega_{n}\right| \in S_{b}} \omega_{n}^{2} \tilde{x}\left(\omega_{n}\right) \tilde{x}\left(-\omega_{n}\right)\right. \\
& \left.-\beta S_{\text {int }}\right]
\end{aligned}
$$

where the $S_{\text {int }}$ is an effective interaction term. In such an equation, the integration over the hard modes is performed in Fourier space,

$$
\mathcal{D} x_{>} \equiv \prod_{\left|\omega_{n}\right| \in S_{b}} d \widetilde{x}\left(\omega_{n}\right)
$$

Equation (27) is formally exact. In the next section, we evaluate the effective action $S_{>}\left[x_{<}(\tau)\right]$ perturbatively. The effective interaction which includes the correction coming from $S_{>}\left[x_{<}\right]$will be referred to as the renormalized effective interaction.

\section{RENORMALIZED EFFECTIVE INTERACTION}

In the previous section, we have seen that the integration over the fast modes generates an additional term in the effective action for the slow modes,

$$
Z(t) \equiv \oint \mathcal{D} x_{<} e^{\left.-\beta S_{e f f}\left[x_{<}(t)\right]\right]-\beta S_{>}\left[x_{<}(\tau)\right]}
$$

where

$$
e^{-\beta S_{>}[x<(\tau)]}=\oint \mathcal{D} x_{>} e^{-\beta t \Sigma_{\left|\omega_{n}\right| \in S_{b}}\left(\gamma \omega_{n}^{2} / 4\right) \widetilde{x}\left(\omega_{n}\right) \widetilde{x}\left(-\omega_{n}\right)} e^{-\beta S_{i n t}} .
$$

In this section we formally perform such an integration. We begin by rewriting $e^{-\beta S_{>}\left[x_{<}(\tau)\right]}$ as

$$
e^{-\beta S_{>}\left[x_{<}(\tau)\right]}=\left\langle e^{\left.-\beta S_{i n t}\right\rangle_{0}},\right.
$$

where the notation $\langle\cdot\rangle_{0}$ denotes the expectation value evaluated in the free theory

$$
S_{>}^{0}\left[x_{>}\right]=t \sum_{\left|\omega_{n}\right| \in S_{b}} \frac{\gamma \omega_{n}^{2}}{4} \tilde{x}\left(\omega_{n}\right) \tilde{x}\left(-\omega_{n}\right) .
$$

To evaluate the matrix element $\left\langle e^{-\beta S_{\text {int }}}\right\rangle$, we represent the

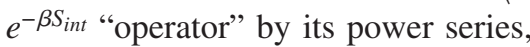




$$
\begin{aligned}
\left\langle e^{\left.-\beta S_{\text {int }}\right\rangle_{0}}\right. & =\sum_{k} \frac{1}{k !}\left\langle\left(-\beta S_{i n t}\right)^{k}\right\rangle_{0} \\
& =\frac{1}{Z_{>}^{0}} \int \mathcal{D} x_{>} \sum_{k} \frac{1}{k !}\left(-\beta S_{\text {int }}\right)^{k} e^{-\beta S_{>}^{0}} .
\end{aligned}
$$

Next, we expand the interaction action $S_{\text {int }}\left[x_{>}+x_{<}\right]$around the slow modes, ${ }^{1}$

$$
\begin{aligned}
-\beta S_{\text {int }}\left[x_{>}+x_{<}\right]= & -\beta \int_{0}^{t} d \tau \frac{\partial V_{e f f}\left[x_{<}(\tau)\right]}{\partial x^{i}} x_{>}(\tau) \\
& -\beta \frac{1}{2} \int_{0}^{t} d \tau \frac{\partial^{2} V_{e f f}\left[x_{<}(\tau)\right]}{\partial x^{i} x^{j}} x_{>}^{i}(\tau) x_{>}^{j}(\tau)+\cdots \\
= & -\beta \int_{0}^{t} d \tau \sum_{k} \frac{1}{k !} V_{i_{1}, \ldots, i_{k}}(\tau) x_{>}^{i_{1}}(\tau) \ldots x_{>}^{i_{k}}(\tau),
\end{aligned}
$$

where $V_{i_{1}, \ldots, i_{k}}(\tau) x_{>}^{i_{1}}(\tau) \ldots x_{>}^{i_{k}}(\tau)$ are vertices with couplings

$$
V_{i_{1}, \ldots, i_{k}}(\tau) \equiv \frac{\partial^{k} V_{e f f}\left[x_{<}(\tau)\right]}{\partial x^{i_{1}} \ldots \partial x^{i_{k}}} .
$$

Notice that each term in the perturbative expansion (35) generates a new vertex, with an increasing power of the $x_{>}(\tau)$ field. The couplings to the fast modes depend implicitly on the time $\tau$, through the slow modes $x_{<}(\tau)$.

By Wick theorem, each term in the series (34) can be related to a Feynman graph with vertexes given by Eq. (36) and propagators given by-see Appendix A,

$$
\begin{aligned}
\left\langle x_{>}^{i}\left(\tau_{1}\right) x_{>}^{j}\left(\tau_{2}\right)\right\rangle_{0} & =\sum_{\left|\omega_{m}\right|,\left|\omega_{n}\right| \in S_{b}} G_{>}^{0 i j}\left(\omega_{n}, \omega_{m}\right) e^{i\left(\omega_{m} \tau_{1}+\omega_{n} \tau_{2}\right)} \\
& =\sum_{\left|\omega_{n}\right| \in S_{b}} \delta_{i j} \frac{2}{\beta \gamma t \omega_{n}^{2}} e^{i \omega_{m}\left(\tau_{2}-\tau_{1}\right)} .
\end{aligned}
$$

The expansion (34) can be reorganized as the exponent of the sum performed over only connected diagrams,

$$
e^{-\beta S_{>}[x<(\tau)]}=e^{\text {(sum over all connected diagrams) }} .
$$

Hence, the path integral [Eq. (27)] for the slow modes can be given the following exact diagrammatic representation:

$$
Z(t) \equiv \oint \mathcal{D} x_{<} e^{\left.-\beta S_{e f f}\left[x_{<}(t)\right]\right\}+(\text { sum over all connected diagrams) }}
$$

Below we give a classification of all the connected diagrams that may give a contribution to the expansion above. First note that all diagrams that involve an odd numbers of fast field vanish thanks to the Wick theorem. We are thus left with the following sets of, a priori nonvanishing, diagrams:

(i) One-particle-reducible 1PR diagrams, namely, diagrams that can be topologically separated into two distinct subdiagrams by cutting one internal fast-mode line (propaga-

\footnotetext{
${ }^{1}$ Throughout all this work, we shall adopt Einstein notation, i.e., the summation over repeated indexes is implicitly assumed.
}

tor): they have the topology of a dumbbell. The simplest examples of dumbbell diagrams are depicted in the upper part of Fig. 2.

The main assumption of this work is the existence of a gap between slow modes and fast modes. Under such assumption all the 1PR diagrams give vanishing contributions. From the physical point of view, this can be understood as a consequence of energy conservation: in order for the total energy flowing through a vertex with a single hard mode to be conserved, at least one of the external modes has to be hard. On the other hand, our working assumption implies that all the modes in the external legs of diagrams are soft. This result can be rigorously proven for all 1PR. As an example, we explicitly compute the upper left diagram of Fig. 2. We have

$$
\begin{aligned}
\frac{1}{2 !}( & \left.-\frac{\beta}{2 !}\right)^{2} \int_{0}^{t} d \tau_{1} \int_{0}^{t} d \tau_{2} V_{i}\left[x_{<}\left(\tau_{1}\right)\right] V_{j}\left[x_{<}\left(\tau_{2}\right)\right] \\
& \times \sum_{\left|\omega_{n}\right| \in S_{b}} \frac{2}{\gamma \beta t} \frac{e^{i \omega_{n}\left(\tau_{2}-\tau_{1}\right)}}{\omega_{n}^{2}} \delta_{i j} .
\end{aligned}
$$

We note that the effective potentials depend smoothly on time, through the periodic functions $x_{<}^{i}(\tau)$. Hence, the terms $V_{i}\left[x_{<}\left(\tau_{1}\right)\right]$ and $V_{j}\left[x_{<}\left(\tau_{2}\right)\right]$ in Eq. (40) can be expressed in terms of their Fourier transform,

$$
\begin{gathered}
V_{i}\left[x_{<}\left(\tau_{1}\right)\right]=\sum_{n} \tilde{V}_{i}\left(\nu_{n}\right) e^{i \nu_{n} \tau_{1}}, \\
V_{j}\left[x_{<}\left(\tau_{2}\right)\right]=\sum_{m} \tilde{V}_{j}\left(\nu_{m}\right) e^{i \nu_{m} \tau_{2}} .
\end{gathered}
$$

This allows to perform the time integrals, which simply yield $t^{2} \delta_{\omega_{n}+\nu_{n}, 0} \delta_{\omega_{n}-\nu_{m}, 0}$. Due to such delta functions, only hard $\nu$ modes survives, which are projected in a term

$$
\propto \sum_{\left|\nu_{n}\right| \in S_{b}} \frac{\beta t}{4 \gamma \nu_{n}^{2}} \frac{1}{\nu_{n}^{2}} \widetilde{V}_{i}\left(\nu_{n}\right) \tilde{V}_{i}\left(-\nu_{n}\right) \approx 0 .
$$

These modes thus yield negligible contributions under the physical assumption of large separation of frequency scales. On the other hand, if one does not assume a separation of time scale, this diagram gives finite contribution and has to be accounted for. Note that this term has the same structure as the first correction which appears when one performs higher-order Trotter expansion [11].

It is not difficult to check that such result holds for all 1PR diagrams, so that we can reduce our effective action to the sum of one-particle-irreducible diagrams, i.e., diagrams that cannot be disconnected by cutting a single internal line. They can be classified in two main groups:

(i) 1PI "daisy" diagrams, namely diagrams with a single vertex. Such diagrams only involve equal-time hard propagators and only give rise to contributions to the renormalized effective action which are local in time: they have the topology of a daisy, hence the name. Examples of daisy diagrams are depicted in the middle part of Fig. 2. It is not difficult to compute a generic daisy diagram with $K$ petals (propagators). It is due to the vertex with $2 K$ hard fields and reads 
$(1 P R)$
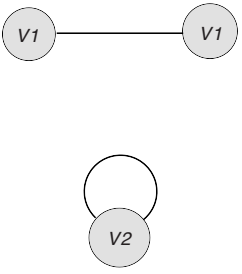

$(1 P I-D)$

$(1 P I-n D)$

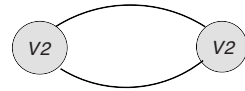

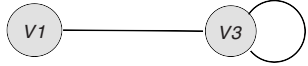
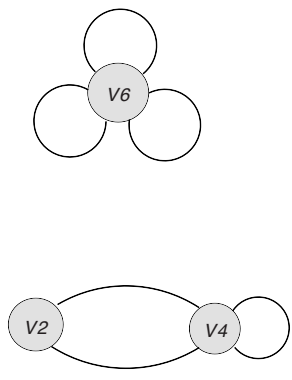

FIG. 2. Examples of connected graphs appearing in the exponent of Eq. (39). The diagrams on the upper part (dumbbell diagrams) are one-particle reducible, while those in the middle and in the bottom are one-particle-irreducible. In particular, those in the middle (daisy diagram) are local in time.

$$
\begin{aligned}
& (2 K-1) ! !\left[-\frac{\beta}{(2 K) !}\right] \int_{0}^{t} d \tau \Delta^{K} V_{e f f}\left[x_{<}(\tau)\right] \\
& \quad \times\left(\frac{2}{\beta \gamma t} \sum_{\left|\omega_{n}\right| \in S_{b}} \frac{1}{\omega_{n}^{2}}\right)^{K},
\end{aligned}
$$

where $\Delta \equiv \delta_{i j} \partial_{i} \partial_{j}$ is the Laplacian operator, and the numerical factor in front is a combinatorial factor. The sum, i.e., the equal-time propagator, can be easily performed by taking the continuum limit $\Sigma_{\omega} \rightarrow \frac{t}{2 \pi} \int d \omega$ that simply yields ${ }^{2}$

$$
\frac{1}{t} \sum_{\left|\omega_{n}\right| \in S_{b}} \frac{1}{\omega_{n}^{2 p}} \rightarrow \frac{1}{\pi} \int_{b \Omega}^{\Omega} \frac{d \omega}{\omega^{2 p}}=\frac{1}{(2 p-1) \pi} \frac{1-b^{2 p-1}}{(b \Omega)^{2 p-1}},
$$

so that we finally obtain

$$
\left(-\frac{\beta}{K !}\right)\left(\frac{D}{\pi} \frac{1-b}{b \Omega}\right)^{K} \int_{0}^{t} d \tau \Delta^{K} V_{e f f}\left[x_{<}(\tau)\right],
$$

where we have reinstated the diffusion coefficient $D=1 / \beta \gamma$. Hence, one can even formally resum all the daisy diagrams into the compact expression,

$$
\sum \text { daisy diagrams }=-\beta \int_{0}^{t} d \tau \exp \left(\frac{D}{\pi} \frac{1-b}{b \Omega} \Delta\right) V_{e f f}\left[x_{<}(\tau)\right] .
$$

(i) 1PI nondaisy diagrams: all other nonlocal diagrams. The simplest examples of such diagrams are depicted in the lower part of Fig. 2. These diagrams generate contributions to the renormalized effective action that are nonlocal in time and give rise to infinite series of local diagrams. For example, the evaluation of the lower left diagram of Fig. 2 yields a contribution of the form,

\footnotetext{
${ }^{2}$ Here for later use we consider a generic even power $2 p$. It is easy to check that the error one makes in considering the continuum limit is of order $\frac{1}{(1-b) N}$ with $N \equiv \Omega t / 2 \pi$.
}

$$
\begin{gathered}
2 \times \frac{1}{2 !}\left(-\frac{\beta}{2 !}\right)^{2} \int_{0}^{t} d \tau_{1} \int_{0}^{t} d \tau_{2} V_{i j}\left[x_{<}\left(\tau_{1}\right)\right] V_{k l}\left[x_{<}\left(\tau_{2}\right)\right] \\
\times \sum_{\left|\omega_{n}\right|,\left|\omega_{m}\right| \in S_{b}}\left(\frac{2}{\gamma \beta t}\right)^{2} \frac{e^{i\left(\omega_{n}+\omega_{m}\right)\left(\tau_{2}-\tau_{1}\right)}}{\omega_{n}^{2} \omega_{m}^{2}} \delta_{i k} \delta_{j l},
\end{gathered}
$$

where the 2 in front is a combinatorial factor. After Fourier transforming the potentials [see discussion below Eq. (40)], the integrals over times yield $t^{2} \delta_{\omega_{m}+\omega_{n}+\nu_{n}, 0} \delta_{\omega_{m}+\omega_{n}-\nu_{m}, 0}$. Hence,

$$
\frac{1}{\gamma^{2}} \sum_{\nu_{n}} \sum_{\left|\omega_{n}\right| \in S_{b}} \tilde{V}_{i j}\left(\nu_{n}\right) \tilde{V}_{i j}\left(-\nu_{n}\right) \frac{1}{\omega_{n}^{2}} \frac{1}{\left(\omega_{n}-\nu_{n}\right)^{2}} .
$$

Now we again make use of the assumption that slow modes and fast modes of physical processes under study are separated by a large gap. Under such assumption we can safely expand the second fraction in the latter expression in power series of slow modes $\nu_{n}$ and rewrite Eq. (49) as higher-timederivative expansion. Let us reintroduce the integral over time as $1=\frac{1}{t} \int_{0}^{t} d \tau \Sigma_{\nu_{m}} e^{i\left(\nu_{n}+\nu_{m}\right) \tau}$ so that powers of $\nu_{n}$ can be traded with time derivative of the potential (note that odd powers vanish upon symmetric sum; in fact they would give rise to total time-derivative terms that are zero upon integration thanks to periodicity in time.) We are thus left with

$$
\begin{aligned}
& \frac{1}{\gamma^{2}} \int_{0}^{t} d \tau V_{i j}\left[x_{<}(\tau)\right]\left[\frac{1}{t} \sum_{\left|\omega_{n}\right| \in S_{b}} \frac{1}{\omega_{n}^{4}}+\frac{3}{t} \sum_{\left|\omega_{n}\right| \in S_{b}} \frac{1}{\omega_{n}^{6}}\left(-\partial_{\tau}^{2}\right)\right. \\
& \left.\quad+\frac{5}{t} \sum_{\left|\omega_{n}\right| \in S_{b}} \frac{1}{\omega_{n}^{8}} \partial_{\tau}^{4}+\cdots\right] V_{i j}\left[x_{<}(\tau)\right] .
\end{aligned}
$$

Sums over hard frequencies can be performed in the continuum limit with the help of formula (45) and time derivatives can be partially integrated in order to rewrite the latter in a more symmetric form

$$
\begin{aligned}
& \frac{1}{\pi \gamma^{2}} \int_{0}^{t} d \tau\left\{\frac{1}{3} \frac{1-b^{3}}{(b \Omega)^{3}}\left[V_{i j}\left(x_{<}\right)\right]^{2}+\frac{3}{5} \frac{1-b^{5}}{(b \Omega)^{5}}\left[V_{i j l}\left(x_{<}\right) \dot{x}_{<}^{k}(\tau)\right]^{2}\right. \\
& \left.+\frac{5}{7} \frac{1-b^{7}}{(b \Omega)^{7}}\left[V_{i j l m}\left(x_{<}\right) \dot{x}_{<}^{l}(\tau) \dot{x}_{<}^{m}(\tau)\right]^{2}+\cdots\right\}
\end{aligned}
$$

The infinite higher-derivative expansion is the legacy of nonlocality in time: such an expansion can be diagrammatically represented as an infinite sum of local (daisylike) diagrams, as depicted in Fig. 3.

It is intuitive to expect that, in the presence of decoupling low and high frequency modes, the higher-derivative terms should be suppressed. In the next section, we shall generalize this statement and present a quantitative method to systematically organize all contributions of the effective action in terms of a perturbative series.

\section{SLOW-MODE PERTURBATION THEORY}

The diagrammatic representation of the path integral given by Eq. (39) is formally exact, but rather useless. In fact, it is obviously impossible to evaluate and resum exactly all the infinitely many Feynman graphs appearing in the exponent. On the other hand, in this section we show that it is 

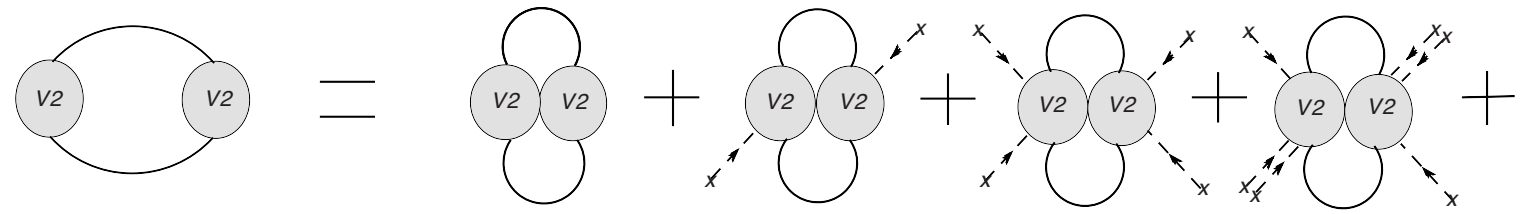

FIG. 3. Diagrammatic representation of the local time-derivative expansion of a nonlocal diagram—Eq. (51). Solid lines are fast-mode propagators, while dashed lines represent a single time derivative acting on the corresponding vertex function.

possible to compute the renormalized effective action $S_{e f f}\left[x_{<}(t)\right]$ to an arbitrary level of precision, by calculating only a finite number of Feynman graphs. This way, the lowfrequency effective theory retains predictive power.

The idea is to exploit the decoupling of the short-time dynamics from the long-time dynamics to organize the sum over all possible graphs as a perturbative expansion. We shall refer to such a systematic evaluation of the renormalized low-frequency effective action as to the slow-mode perturbation theory.

The first step in the construction of our perturbation series is to identify all the dimensionless combinations of the physical quantities which appear in the Feynman graphs contributing to Eq. (39), evaluated in stationary phase approximation. Let us first define the quantities

$$
\begin{gathered}
\bar{V} \equiv \frac{1}{t}\left|\int_{0}^{t} d \tau V\right|, \quad \bar{V}_{2 m}^{k} \equiv \frac{1}{t \bar{V}}\left|\int_{0}^{t} d \tau \Delta^{m} V\right| \sim k^{2 m}, \\
\bar{V}_{2 m}^{\omega} \equiv \frac{1}{t \bar{V}^{2}} \int_{0}^{t} d \tau\left(\partial_{\tau}^{m} V\right)^{2} \sim \omega_{<}^{2 m},
\end{gathered}
$$

where $k$ is the typical wave vector on the spatial Fourier transform of $V_{e f f}(x)$ and $\omega_{<}$is the typical frequency in temporal Fourier transform of $V_{\text {eff }}\left(x_{<}(\tau)\right)$.

Using these combinations, we can thus construct the following dimensionless combinations:

$$
\alpha_{1} \equiv \frac{\beta \bar{V}}{b \Omega}, \quad \alpha_{2} \equiv \frac{k^{2} D}{b \Omega}, \quad \alpha_{3} \equiv \frac{\omega_{<}}{b \Omega} .
$$

We are interested in describing the dynamics of physical systems for which each of these parameters can be considered small. In order to illustrate the physical interpretation of the condition $\alpha_{1} \ll 1$, we observe that the probability for the system to remain in the same configuration $x$, during an elementary time interval $d t$ is

$$
P(x, t+d t \mid x, t) \propto \frac{1}{(d t)^{d / 2}} e^{-\beta V_{e f f}(x) d t} .
$$

Hence, the combination $\beta V_{\text {eff }}$ represents ${ }^{3}$ the typical time scale associated to local conformational changes, and the condition $\alpha_{1} \ll 1$ expresses the condition that the time spent on average by the system in each configuration is large compared to the elementary short-time scale, $d t \sim \frac{1}{b \Omega}$.

\footnotetext{
${ }^{3}$ Notice that, in the small temperature limit, $V_{\text {eff }}(x)$ becomes positive definite. Thus, $P(x, t+d t \mid x, t)$ decays exponentially with $\beta V_{\text {eff }}$ in the time interval $d t$.
}

The condition $\alpha_{2} \ll 1$ implies that the effective potential varies over length scales which are large, compared with the mean distance covered by Brownian motion in an elementary time interval $d t$. Finally, the condition $\alpha_{3} \ll 1$ implies that the typical slow-mode frequencies are small compared to the ultraviolet cutoff, which is of the order of the inverse of the elementary time interval $d t$.

It is easy to see that any local diagram in the expansion of the renormalized effective action comes about with integer powers of these coefficients, when compared to the tree-level effective action. In particular, any diagram composed by $r$ vertices of $M_{1}, \ldots, M_{r}$ hard fields will involve $M=\sum_{i=1}^{r} M_{i}$ spatial derivatives and $\frac{M}{2}$ propagators each of which yields a power of $\frac{1}{b \Omega}$. Finally, each additional vertex yields a power of $\beta \bar{V}$ and each time derivative yields a power of $\omega$. So, the above diagram, at the lowest level in time derivatives will be of order $\alpha_{1}^{r-1} \alpha_{2}^{M / 2}$ with respect to the tree-level expression. Higher time derivative terms will add powers $\alpha_{3}$. It is thus natural to define a degree of slowness $L$ for a local diagram, given by

$$
L(\text { Feynman diagram })=N_{v}-1+N_{\tau}+\frac{N_{x}}{2},
$$

where $N_{v}$ is the number of vertices, $N_{\tau}$ the number of time derivatives and $N_{x}$ the number of spatial derivatives. The definition in Eq. (55) is normalized in such a way that $L($ tree $)=0$.

It is easy to check that the degree of slowness $L$ corresponds to the power of $\frac{1}{b \Omega}$ of the local diagrams. Note also

$(L=1)$
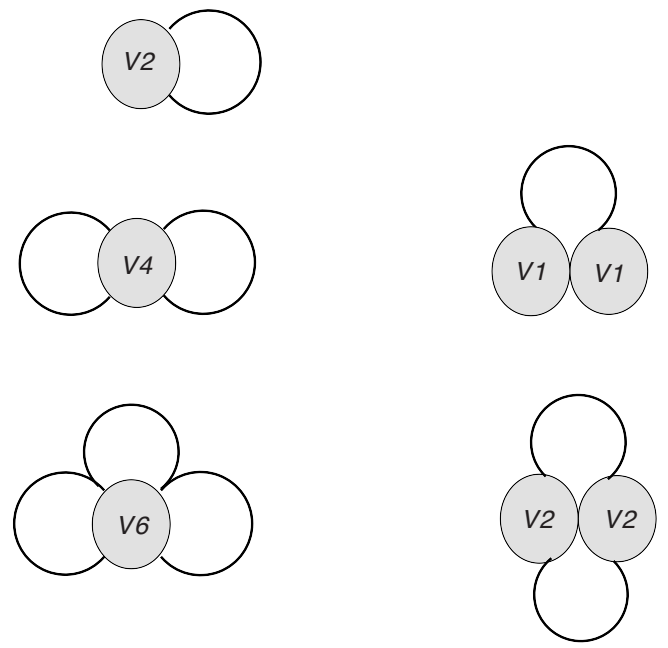

FIG. 4. Examples of the diagrams with the lowest degree of slowness, up to $L=3$. 
that for daisy diagrams and for all other diagrams where $N_{\tau}=0$, the degree $L$ is nothing but the number of loops. One can thus easily write down and compute the finite set of local diagrams that renormalize the effective action up to a fixed (yet arbitrary) level of precision $L_{\text {max }}$. Let us consider a few simple examples.

(i) $L \leq 1$ corresponds to a single daisy diagram with $L$ $=1, N_{v}=1$ and $N_{x}=2$, represented in the left panel of Fig. 4 . The expression of this diagram is given by Eq. (46) and gives a correction to the effective action of the form

$$
S_{>}\left[x_{<} ; L \leq 1\right]=\frac{D}{\pi} \frac{1-b}{b \Omega} \int_{0}^{t} d \tau \Delta V_{e f f}\left[x_{<}(\tau)\right] .
$$

(i) $L \leq 2$ corresponds to two further diagrams, one daisy diagrams with either $N_{x}=4$ and the two-vertex local diagram with $N_{x}=2$ and no time derivatives. This latter however is
$1 \mathrm{PR}$ and gives no contribution. We are thus left with the corrections

$$
\begin{aligned}
S_{>}\left[x_{<} ; L \leq 2\right] & =\frac{D}{\pi} \frac{1-b}{b \Omega} \int_{0}^{t} d \tau \Delta V_{e f f}\left[x_{<}(\tau)\right] \\
& +\frac{1}{2}\left(\frac{D}{\pi} \frac{1-b}{b \Omega}\right)^{2} \int_{0}^{t} d \tau \Delta^{2} V_{e f f}\left[x_{<}(\tau)\right]
\end{aligned}
$$

(ii) $L \leq 3$ corresponds to two further diagrams, one daisy diagrams with $N_{x}=6$ and the two-vertex local diagram with $N_{x}=4$ and no time derivatives. This latter can be simply read off from Eq. (51). Hence

$$
\begin{aligned}
& S_{>}\left[x_{<} ; L \leq 3\right]=\frac{D}{\pi} \frac{1-b}{b \Omega} \int_{0}^{t} d \tau \Delta V_{e f f}\left[x_{<}(\tau)\right]+\frac{1}{2 !}\left(\frac{D}{\pi} \frac{1-b}{b \Omega}\right)^{2} \int_{0}^{t} d \tau \Delta^{2} V_{e f f}\left[x_{<}(\tau)\right] \frac{1}{3 !}\left(\frac{D}{\pi} \frac{1-b}{b \Omega}\right)^{3} \int_{0}^{t} d \tau \Delta^{3} V_{e f f}\left[x_{<}(\tau)\right] \\
& -\frac{\beta D^{2}}{3 \pi} \frac{1-b^{3}}{(b \Omega)^{3}} \int_{0}^{t} d \tau\left\{\partial_{i} \partial_{j} V_{e f f}\left[x_{<}(\tau)\right]\right\}^{2}
\end{aligned}
$$

that involves in the last term the trace of the square of Hessian of the tree-level potential $\left[\partial_{i} \partial_{j} V\left(x_{<}\right)\right]^{2}=\operatorname{Tr} \mathcal{H}_{V}^{2}$. In order to see the first time derivatives appearing into the renormalized effective action we need to consider $L \leq 5$ where, along with several other corrections, we have the correction coming from the second term in Eq. (51) that yields

$$
-\frac{3 \beta D^{2}}{5 \pi} \frac{1-b^{5}}{(b \Omega)^{5}} \int_{0}^{t} d \tau \operatorname{Tr} \dot{\mathcal{H}}_{V}^{2}
$$

that can be also recast as a correction of the kinetic action

$$
\beta \gamma \int_{0}^{t} d \tau\left[\frac{1}{4} \delta_{k l}-\frac{3 \beta^{2} D^{3}}{5 \pi} \frac{1-b^{5}}{(b \Omega)^{5}} \partial_{i} \partial_{j} \partial_{k} V \partial_{i} \partial_{j} \partial_{l} V\right] \dot{x}^{k} \dot{x}^{l} .
$$

Some comments on the results obtained in this section are in order. First of all, we emphasize that the effective interactions have been derived under the assumption that the modes which are relevant for the long-time dynamics vary over time scales much longer than that of the fast modes, which enter in the loop diagrams. This is the crucial assumption of all renormalization group approaches. Our results confirm the intuitive picture that if one adopts a low "time-resolution power," then the effective interactions generated by the ultraviolet modes can be regarded as instantaneous. This is in fact general property of renormalization group theory, which is preserved to any order in the perturbative expansion. Finally, we note that the correction terms generated by the integration over the fast modes are suppressed, in the small temperature limit.

\section{RENORMALIZATION GROUP IMPROVED MONTE CARLO}

The usefulness of the renormalization procedure resides in the fact that it gives rise to an effective theory, in which the largest frequency scale is lowered form $\Omega$ to $b \Omega$. Equivalently, the shortest time scale is increased form $\Delta t$ to $\frac{1}{b} \Delta t$. By construction, in the regime of decoupling of fast and slow modes, the probability density generated by the new slowmode effective theory must be the same as that of the original (i.e., tree-level) theory. In this section, we show how it is possible to use the slow-mode effective theory to develop improved MC algorithms for the time evolution of the probability density $P(x, t)$, in which the elementary time step used to propagate the configurations is increased by a factor $1 / b$.

The starting point of the MC approach [17] is to write the probability of observing the system in configuration $x$ at time $t$ in terms of the Green's function of the Fokker-Planck equation $G\left(x, t \mid x_{i}, t_{i}\right)$,

$$
P(x, t)=\int d x_{i} G\left(x, t \mid x_{i}, t_{i}\right) \rho_{0}\left(x_{i}\right)
$$

where $\rho_{0}(y)$ is the density of states at the initial time.

One then uses Trotter's formula to write the transition probability as a sequence of intermediate elementary propagation steps, 


$$
P(x, t)=\int \prod_{k=0}^{N-1} d y_{k} G\left(y_{k+1}, t_{k+1} \mid y_{k}, t_{k}\right) \rho_{0}\left(x_{i}\right) \quad\left(y_{0}=x_{i}, y_{N}=x\right) .
$$

If a sufficiently large number of intermediate steps $N$ is adopted, then the time steps $\Delta t=t_{k+1}-t_{k}$ can be considered infinitesimal and the (un-normalized) transition probability $G\left(y_{k+1}, t_{k+1} \mid y_{k}, t_{k}\right)$ can be calculated analytically.

$$
\begin{aligned}
G(y & +d y, t+\Delta t \mid y, t) \\
& =\text { const. } \times e^{-\beta\left((\gamma / 4)(d y / \Delta t)^{2} \Delta t+(1 / 2)(d y / \Delta t) \cdot \nabla U(y)\right) \Delta t} e^{-\beta V_{e f f}(y) \Delta t} .
\end{aligned}
$$

"Completing the square" in the first exponent, one finds

$$
\begin{aligned}
& G(y+d y, t+\Delta t \mid y, t) \\
& \quad=\text { const. } \times e^{-(1 / 4 D \Delta t)[d y+(\Delta t / \gamma) \nabla U(y)]^{2}+(\beta / 4 \gamma)(\nabla U)^{2} \Delta t} e^{-\beta V_{e f f}(y) \Delta t} .
\end{aligned}
$$

Now and recalling the definition of the effective potential [Eq. (8)] in the second exponent, this Green's function can be written as

$$
\begin{aligned}
G(y & +d y, t+\Delta t \mid y, t) \\
& =\text { const. } \times e^{-(1 / 4 D \Delta t)[d y+(\Delta t / \gamma) \nabla U(y)]^{2}} e^{(1 / 2 \gamma) \nabla^{2} U(y) \Delta t}
\end{aligned}
$$

In the MC algorithm, one starts from a set of initial system's configurations, sampled according to he distribution $\rho_{0}\left(x_{i}\right)$. Such an ensemble is evolved in time, according to the following procedure. Each configuration is propagated for an elementary time interval $\Delta t$, by sampling from the Gaussian

$$
e^{-(1 / 4 D \Delta t)[d y+(\Delta t / \gamma) \nabla U(y)]^{2}}
$$

in Eq. (65). Such a configuration is then reweighted according to the factor

$$
\mathcal{W}(y)=e^{(1 / 2 \gamma) \nabla^{2} U(y) \Delta t} .
$$

The iteration of such a procedure for many consecutive elementary propagations gives rise to a set of diffusive trajectories, called walkers. In the so-called diffusion MC algorithm, the term $\mathcal{W}$ is used to replicate or annihilate the walkers. The ensemble of configurations obtained according to this procedure is distributed according to the probability density (61).

For the MC algorithm to be efficient, the fluctuations in the statistical weight of the walkers-or, equivalently, in the number of walkers - should remain small, throughout the entire time evolution. This condition is verified if the factor $\mathcal{W}(y)$ is always of order one. Note however that this term tends to enhance (suppress) the weight of configurations in the vicinity of the local minima (maxima) of $U(y)$, where the Laplacian is positive (negative). Hence, if the energy landscape varies very rapidly in space, then the fluctuations in the statistical weights-or in the number of walkers-will in general be large, unless the elementary time step $\Delta t$ is chosen very small. This feature represents a limiting factor of $\mathrm{MC}$ simulations, which makes the sampling of the probability density at large times very computationally expensive.

Clearly, the elementary propagation time step $\Delta t$ is the shortest time scale in the simulation. On the other hand, in the slow-mode effective theory one integrates out the dynamics in the time scale range $(\Delta t, 1 / b \Delta t)$. Hence, we expect that by taking into account of the corrections associated to the renormalized effective interaction it is possible to perform MC simulations in which the integration time step $\Delta t$ is chosen a factor $1 / b$ larger.

In practice, the effective slow-mode theory introduces a correction in the reweighting-or branching. To order $L=1$ one has

$$
\mathcal{W}_{L=1}(y)=e^{(1 / 2 \gamma) \nabla^{2} U(y) \Delta t} \times e^{-(D / \pi)((1-b) / b \Omega) \nabla^{2} V_{e f f}(y) \Delta t}
$$

Notice that this expression contains a factor of the inverse frequency cutoff $1 / \Omega$ in the exponent. Such a term is proportional to the elementary time step $\Delta t$. The corresponding proportionality factor reads $2 \pi$ only for periodic path integral. For a generic initial value $\mathrm{MC}$ one can write in general

$$
\Omega=\kappa \frac{2 \pi}{\Delta t},
$$

where the constant $\kappa$ is to be determined from simulations. Hence, we obtain

$$
\mathcal{W}_{L=1}(y)=e^{2 \nabla^{2} U(y) \Delta t} \times e^{-\kappa\left(D / 2 \pi^{2}\right)((1-b) / b) \nabla^{2} V_{e f f}(y) \Delta t^{2}}
$$

The unknown constant $\kappa$ can be determined by matching the results obtained by running a short simulation in the tree-level theory-i.e., using an integration step $\Delta t$ and the tree-level weighting term [Eq. (67)]—with those obtained in the effective theory-i.e., using an integration step $1 / b \Delta t$ and the renormalized weighting term [Eq. (70)]. In the regime of decoupling of fast and slow modes, once the matching has been done, the two algorithms must generate the same evolution for the probability density at any later times. We emphasize that, since the expressions (56)-(58) have been derived in the continuum limit, they are independent on the choice of the prescription adopted to define the stochastic calculus. On the other hand, the discretized expressions (67) and (70) are based specifically on the choice of the Stratonovich prescription.

In the next session, we shall provide an example which illustrates how this procedure works in practice and show that the fundamental and the effective theory do indeed generate the same long-time stochastic dynamics.

\section{ILLUSTRATIVE EXAMPLE}

In order to illustrate how the renormalization of the effective interaction works in a simple example, let us consider the dynamics of a point particle, diffusing in a rugged asymmetric harmonic oscillator,

$$
U(x)=h_{1} x^{2}+h_{2} x+h_{3} \sin (w x),
$$

with $h_{1}=2, h_{2}=1, h_{3}=1, w=4$. The viscosity coefficient is set to $\gamma=5$ and inverse temperature to $\beta=5$. Note that this 
potential has been chosen in such a way that the average value of $x$ at thermal equilibrium is nonvanishing. The corresponding $L=0, L=1$, and $L=2$ effective potentials read,

$$
\begin{aligned}
V_{e f f}^{L=0}(x)= & \frac{1}{4 \gamma}\left\{\left[h_{2}+2 h_{1} x+h_{3} w \cos (w x)\right]^{2}\right. \\
& \left.-\frac{2}{\beta}\left[2 h_{1}-h_{3} w^{2} \sin (w x)\right]\right\} \\
V_{e f f}^{L=1}(x)= & V_{e f f}^{L=0}(x)+\frac{1}{4 \gamma}\left(\frac { ( 1 - b ) D } { b \Omega \pi } \left\{-2 h_{3} w^{3} \cos (w x)\left[h_{2}\right.\right.\right. \\
& \left.+2 h_{1} x+h_{3} w \cos (w x)\right]-\frac{2 h_{3} w^{4} \sin (w x)}{\beta} \\
& \left.\left.+2\left[-2 h_{1}+h_{3} w^{2} \sin (w x)\right]^{2}\right\}\right) \\
V_{e f f}^{L=2}(x)= & V_{e f f}^{L=0}(x)+V_{e f f}^{L=1}(x)+\frac{D^{2}(1-b)^{2}}{4 b^{2} \beta \gamma \Omega \pi^{2}} h_{3} w^{4}\left[\beta w \left(h_{2}\right.\right. \\
& \left.+2 h_{1} x\right) \cos (w x)+4 \beta h_{3} w^{2} \cos (2 w x) \\
& \left.+\left(8 \beta h_{1}+w^{2}\right) \sin (w x)\right] .
\end{aligned}
$$

The diffusion Monte Carlo algorithm used in our numerical simulations is presented in the Appendix B. The factor $\Omega$, which appears in the $L=1, L=2$ improvement terms was determined from the time interval $\Delta t$ using Eq. (69). The proportionality constant $\kappa$ in Eq. (70) was determined once and for all, by matching the result of $\langle x(t)\rangle$ of the unimproved (i.e., $L=0$ ) simulations after 10 integration time steps with $\Delta t=0.01$, with those of the RG-improved (i.e., $L$ $=1, L=2$ ) MC simulations after a single elementary time step, with $\Delta t^{\prime}=0.1$. We found $\kappa=0.35$, with no appreciable difference between the $L=1$ and $L=2$ estimates.

Let us now discuss the results of our simulations. We begin by analyzing the effects of accounting for the factor $\mathcal{W}(x)$ defined in Eq. (67), in numerical MC simulations. Figure 5 shows the average position, once the system has attained thermal equilibrium, obtained by diffusion MC simulations with and without branching the walkers according to $\mathcal{W}(x)$. We recall that neglecting such a term is equivalent to simulating the dynamics in the Ito calculus, while the branching is expected to improve the time discretization to order $\Delta t^{2}$. Indeed, our results show that, when one chooses small discretization steps, the two approaches are consistent with each other and yield the exact equilibrium averagewhich was computed directly from the Boltzmann distribution. On the other hand, at large discretization steps, accounting for the factor $\mathcal{W}$ significantly improves the result. The same discussion can be trivially repeated in simulations in which the factor $\mathcal{W}(x)$ is interpreted as a reweighting term, while the number of walkers is held constant.

We now discuss the use of our effective theory to simulate the stochastic dynamics, using large time steps. Figure 6 shows the time evolution of the average particle position at time $t$, computed using a small discretization time step- $\Delta t$ $=0.01-$ and a large discretization step $-\Delta t^{\prime}=0.1$. The two curves obtained in the original-i.e., tree-level-theory are

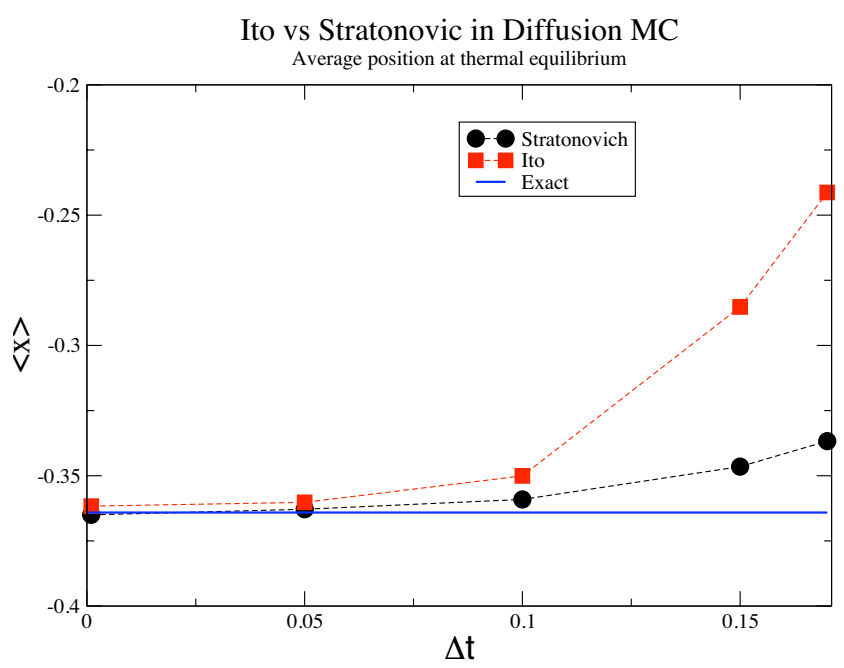

FIG. 5. (Color online) Average position at thermal equilibrium, obtained from diffusion MC simulations with (circles) and without (squares) the branching factor $\mathcal{W}(t)$ of Eq. (67), for different values of the discretization time step $\Delta t$. Errors are smaller than the symbols.

compared with the results of the effective theory at order $L$ $=1$ and $L=2$, which were obtained using an integration time step which was one order of magnitude larger, $\Delta t^{\prime}=0.1$.

The time evolution of the observable $\langle x(t)\rangle$, obtained in the tree-level theory using large integration time steps (squares) is inconsistent with the same quantity obtained using small time steps $\Delta t=0.01$ (circles). This is expected, because for $\Delta t=0.1$ the numerical simulations of the tree-level theory start to be affected by significant discretization errors-see Fig. 5.

The results of simulations with large discretization time steps are significantly improved if one uses the effective theory, already at order $L=1$ (diamonds). At order $L=2$ the

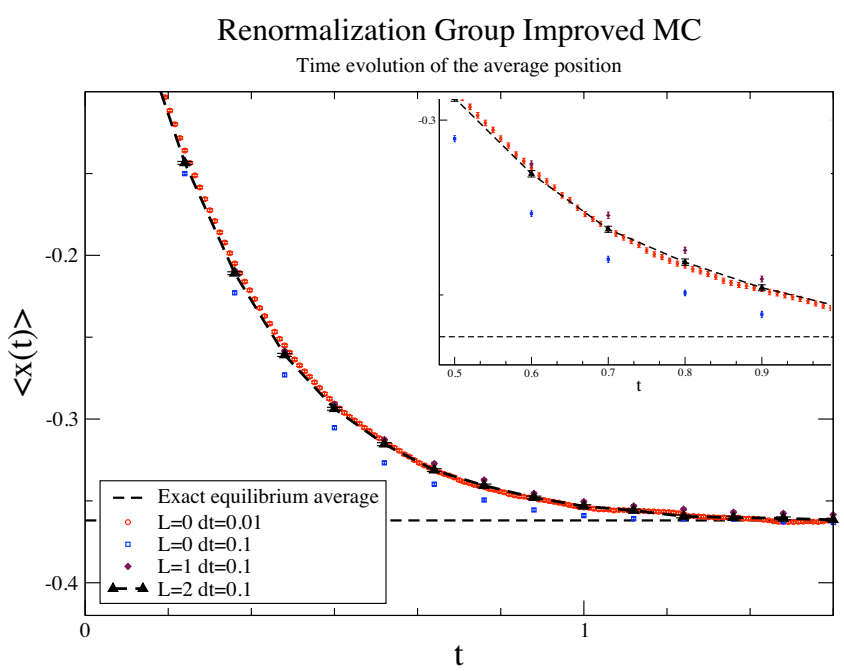

FIG. 6. (Color online) The average position of the particle at time $t$, computed in the tree-level theory (circles, $\Delta t=0.01$ for $L$ $=0$ ), and in the effective theory (squares $\Delta t=0.1$ for $L=0, L=1$ and $L=2$ ). The inset displays a part of the same curve, on a larger scale. Statistical errors are smaller than the symbols. The $\Delta t=0.1, L=2$ cannot be distinguished from the $\Delta t=0.01, L=0$ curve. 
dynamics of the tree-level theory simulated at $\Delta t=0.01$ is indistinguishable from the dynamics of the effective theory simulated with $\Delta t^{\prime}=0.1$ (triangles). These results show that the hard-mode dynamics in the short-time range from 0.01 to 0.1 has been correctly taken into account by means of the renormalized effective interaction. As a consequence, the use of the effective theory allows to obtain very accurate predictions, using larger time steps.

\section{LONG-TIME DYNAMICS OF MOLECULAR SYSTEMS}

The improvement of the MC algorithm based on our effective theory is expected to be most efficient when the gap between the slow and the fast modes is very large. In fact, in this regime, the slow-mode perturbation theory remains reliable even when one integrates out a large frequency shell, i.e., when $b \ll 1$. Hence, in this case, by RG improvement it is possible to simulate the time evolution using elementary time steps $\Delta t^{\prime}$ which are significantly larger than the original elementary time step $\Delta t$, which would be used in the usual (unimproved) $\mathrm{MC}$ algorithm.

A natural application of the RG-improved MC is the investigation of the long-time dynamics of macromolecules, for which standard MD or MC algorithms can be extremely computationally expensive. Hence, it is interesting to address the question of what is the typical range of reliability of the slow-mode perturbation theory for a typical molecular interaction, at room temperature. To this end, let us consider the overdamped diffusion at temperature $300 \mathrm{~K}$ of two molecules of mass $m=30 \mathrm{amu}$, interacting through a van der Waals potential,

$$
U(r)=4 \epsilon\left[\left(\frac{\sigma}{r}\right)^{12}-\left(\frac{\sigma}{r}\right)^{6}\right],
$$

where $\epsilon=4 \mathrm{KJ} / \mathrm{mol}$ and $\sigma=0.3 \mathrm{~nm}$. A typical value for the viscosity coefficient for a molecule in its solvent (e.g., an amino acid in water) is $\gamma \sim 2 \times 10^{3} \mathrm{amu} \mathrm{ps}^{-1}$. The typical time steps used in the numerical integration of the Langevin Eq. (2) are of the order $\Delta t \simeq 10^{-3}-10^{-2}$ ps.

The tree-level effective interaction associated to the potential (75) is

$$
V_{\text {eff }}(r)=\frac{1}{4 \gamma}\left\{\left[24 \epsilon \frac{\sigma^{6}}{r^{7}}\left(1-2 \frac{\sigma^{6}}{r^{6}}\right)\right]^{2}-\frac{8 \epsilon}{\beta} \frac{\sigma^{6}}{r^{8}}\left[156 \frac{\sigma^{6}}{r^{6}}-42\right]\right\} .
$$

This function and the corresponding $L=1$ and $L=2$ renormalized effective interactions are plotted in Fig. 7 for $\kappa=1$. This plot shows that, for a realistic set of parameters, the perturbative expansion remains reliable even when one integrates out a very large shell of modes, with $b \sim 10^{-2}$. This fact suggests that the ultraviolet dynamics is essentially free brownian motion, while the long-time dynamics is dominated by very low-frequency modes, and is driven by the force field. This fact has remarkable consequences on practical numerical simulations. It implies that by using the renormalized effective potential, it should be possible to adopt integration time steps which are about $10^{2}$ time larger

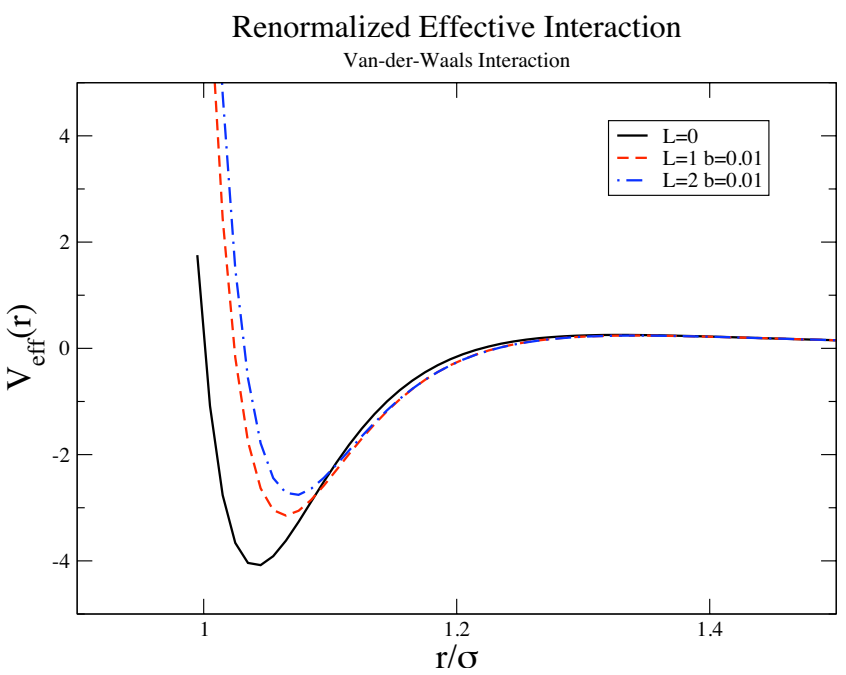

FIG. 7. (Color online) The tree-level, $L=1$ and $L=1$ renormalized effective potential $V_{e f f}(r)$ for the van der Waals interaction Eq. (75), obtained integrating out the modes in the shell $S_{b}=(b \Omega, \Omega)$ with $\Omega=2 \pi / 0.01$ ps and $b=0.01$.

than those required to simulate the dynamics in the original tree-level theory.

\section{CONCLUSIONS}

In this work, we have presented a new approach to the problem of investigating the long-time out-of-equilibrium dynamics of multidimensional systems obeying Langevin dynamics. In the presence of decoupling of time scales, the methods based on the direct integration of the Langevin equation (MD) or on the time propagation of the FokkerPlanck probability density (MC) are usually inefficient, because a significant amount of computational time in invested to simulate uninteresting fast stochastic fluctuations.

We have shown that the decoupling of time scales which limits MD and MC approach can in fact be exploited to perform analytically the average over the short-time stochastic fluctuations. After the integration over the fast modes has been performed, one obtains an effective theory which describes directly the relevant dynamics, with a lower time resolution. In such an effective theory, the effective action in the path integral receives corrections, which account for the ultraviolet physics which is cutoff. We have developed a rigorous scheme which allows to organize such corrections in term of a perturbative series in which the expansion parameters are the ratio between the soft frequency scales and the hard frequency scale $b \Omega$. Hence, subleading terms in the perturbative expansion come with higher inverse powers of the hard scales $b \Omega$ and become irrelevant in the limit in which the decoupling of fast and slow modes is very large.

The Feynman diagrams which have to be calculated to obtain the corrections to any given order in this perturbation theory can be identified from their degree of slowness

$$
L(\text { Feynman diagram })=N_{v}-1+N_{\tau}+\frac{N_{x}}{2} .
$$

Diagrams with degree of slowness $L$ generate corrections proportional to $1 /(b \Omega)^{L}$. In particular, we have found that 
the leading-order correction (i.e., $L=1$ ) is proportional to the Laplacian of the effective potential $V_{\text {eff }}$,

$$
S_{>}\left[x_{<}\right] \simeq \frac{D}{\pi} \frac{1-b}{b \Omega} \int_{0}^{t} d \tau \Delta V_{e f f}\left[x_{<}(\tau)\right] .
$$

At the next-to-leading order, a term containing fourth-order derivatives appears,

$$
\begin{aligned}
S_{>}\left[x_{<}\right] \simeq & \frac{D}{\pi} \frac{1-b}{b \Omega} \int_{0}^{t} d \tau \Delta V_{e f f}\left[x_{<}(\tau)\right] \\
& +\frac{1}{2}\left(\frac{D}{\pi} \frac{1-b}{b \Omega}\right)^{2} \int_{0}^{t} d \tau \Delta^{2} V_{e f f}\left[x_{<}(\tau)\right] .
\end{aligned}
$$

On the other hand, a space-dependent, tensor correction to the diffusion coefficient appears only as a higher-order effect $(L=5)$. It is important to stress the fact that, in the present approach, the ultraviolet cutoff $\Omega$ (or, equivalently, the shorttime scale $\Delta t$ ) is kept finite at all stages. Upon taking the continuum limit $\Delta t \rightarrow 0$, all the correction terms in the effective theory vanish and one recovers the original theory, defined by the effective Schrödinger equation (6).

The main usefulness of such an effective theory resides in the fact that it can be used to develop an improved MC approach, to compute the long-time evolution of the FokkerPlanck probability. In this work, we focused specifically on the implementation of the improved MC approach based on the Stratonovich calculus and leave to future study the comparison between our computation and the corresponding Ito result. The elementary time steps used in the RG improved $\mathrm{MC}$ algorithm are a factor $1 / b$ larger those of the MC algorithm for the underlying tree-level theory. Since the dynam- ics in the time range $(\Delta t, 1 / b \Delta t)$ is averaged analytically, the RG improved MC algorithm avoids investing computational time in simulating the fast-mode dynamics associated to local Brownian motion.

In the specific case of molecular interactions at room temperature, we have shown that the perturbative approach remains reliable even when one integrates large frequency shells, with $b \simeq 0.01$. This feature suggests that, by using the effective theory, it is possible to simulate time intervals which can be up to a factor $\sim 100$ longer than in the usual MC approach.

\section{ACKNOWLEDGMENTS}

We thank A. Szabo for reading the manuscript and making useful comments. Discussions with P. Armani, M. Sega, F. Pederiva, P. Verrocchio, and G. Garberoglio were also useful. P.F. acknowledges financial support from C.N.R.S., during his permanence at the I.Ph.T. of C.E.A. and from I.N.F.N., under the AD31 scientific initiative. The work of O.C. was partly supported by the Italian MIUR-PRIN Contract No. 20075ATT78.

\section{APPENDIX A: PROPAGATOR OF THE FAST MODES}

Here we derive free fast-mode propagator $G_{>}^{0}\left(\omega_{n}, \omega_{m}\right)$ appearing in the diagrams, using the standard source technique. We first add a source term to $Z_{>}^{0}$,

$$
\begin{aligned}
Z_{>}^{0} & \rightarrow Z_{>}^{0}\left[\eta\left(\omega_{n}\right)\right] \\
& =\int \mathcal{D} x_{>} e^{-\beta t \Sigma_{\left|\omega_{n}\right| \in S_{b}}\left[\left(\gamma \omega_{n}^{2} / 4\right) \widetilde{x}\left(\omega_{n}\right) \tilde{x}\left(-\omega_{n}\right)+\widetilde{x}\left(\omega_{n}\right) \eta\left(-\omega_{n}\right)\right]},
\end{aligned}
$$

$$
=\int \mathcal{D} x_{>} e^{-(\beta t / 2) \Sigma_{\left|\omega_{n}\right| \in S_{b}}\left\{\gamma \omega^{2} / 2(\gamma / 2) \omega(2 / n)\left[\tilde{x}\left(\omega_{n}\right)+\left(2 / \gamma \omega_{n}^{2}\right) \eta\left(\omega_{n}\right)\right]\left(\widetilde{x}\left(-\omega_{n}\right)+\left(2 / \gamma \omega_{n}^{2}\right) \eta\left(-\omega_{n}\right)\right)+\left(2 / \gamma \omega_{n}^{2}\right)\left(\eta\left(\omega_{n}\right) \eta\left(\omega_{-n}\right)\right)\right\} .}
$$

Then, we functionally differentiate twice with respect to the source,

$$
G_{>}^{0}\left(\omega_{n}, \omega_{m}\right)=\lim _{\eta \rightarrow 0} \frac{1}{(\beta t)^{2}} \frac{\delta}{\delta \eta\left(-\omega_{n}\right)} \frac{\delta}{\delta \eta\left(-\omega_{m}\right)} \exp \beta t \sum_{\left|\omega_{n}\right| \in S_{b}} \eta\left(\omega_{n}\right) \eta\left(-\omega_{n}\right)=\frac{2}{\beta \gamma t \omega_{n}^{2}} \delta_{\omega_{m}+\omega_{n}, 0} .
$$

Note that since the zero mode belongs to the slow modes part of the kinetic action, the kinetic operator for the fast modes is never singular and can be inverted without troubles.

\section{APPENDIX B: DIFFUSION MONTE CARLO ALGORITHM}

Our numerical study were performed using the following diffusion Monte Carlo algorithm:

(1) A ensemble of $N_{w}=18000$ initial configurations $\left\{x_{1}(t\right.$ $\left.=0), \ldots, x_{N_{w}}(t=0)\right\}$ was generated by sampling from a narrow Gaussian distributions of width $\sigma=0.01$, centered at the origin $x=0$. Each of such positions represents the starting point of a walker.

(2) A new set of $N_{w}$ configurations was obtained by evolving the initial points for an elementary interval $\Delta t$, according to the Langevin dynamics in the Ito calculus,

$x_{l}(t+\Delta t)=x_{l}(t)-\frac{\Delta t}{\gamma} \frac{d}{d x} U\left[x_{l}(t)\right]+\Delta t \eta, \quad l=1, \ldots, N_{w}$.

$\Delta t \eta$ represents the usual Brownian diffusion term, which was performed by sampling from a Gaussian of width $\sigma^{2}$ $=\frac{2}{\beta \gamma} \Delta t$, centered at the origin. 
(3) For each walker, we generated a random number $\xi$ $\in[-0.5,0.5]$ and we made $N_{c}$ copies of the walker, where $N_{c}$ is the integer part of $\mathcal{W}[x(t+\Delta t)]+\xi$. Hence, for $N_{c}=0$ the walker was aborted, for $N_{c}=1$ the walker was left unchanged, while for $N_{c}>1$ the walker gave raise to descendents, which then propagated independently from the progenitor. The integration time step $\Delta t$ was chosen in such a way that the relative fluctuations in the population of walkers were only occasionally exceeding $10 \%$.

(4) The steps 2-3 were iterated for many integration time steps.

(5) The quantity $\langle x(t)\rangle$ was obtained from the mean over the configurations of the walkers. The statistical error was estimated from the variance.
[1] V. J. Pande, I. Baker, J. Chapman, S. P. Elmer, S. Khaliq, S. M. Larson, Y. M. Rhee, M. R. Shirts, C. D. Snow, E. J. Sorin, and B. Zagrovic, Biopolymers 68, 91 (2003).

[2] R. Zwanzig, J. Chem. Phys. 33, 1338 (1960); Phys. Rev. 124, 983 (1961); H. Mori, Prog. Theor. Phys. 33, 423 (1965).

[3] W. Just, H. Kantz, C. Rodenbeck, and M. Helm, J. Phys. A 34, 3199 (2001).

[4] J. Balakrishnan, Phys. Rev. E 73, 036206 (2006).

[5] G. Jayachandran, V. Vishal, and V. S. Pande, J. Chem. Phys. 124, 164902 (2006); G. Jayachandran, V. Vishal, A. E. Garca, and V. S. Pande, J. Struct. Biol. 157, 491 (2007).

[6] J. D. Chodera, W. C. Swope, J. W. Pitera, and K. A. Dill, Multiscale Model. Simul. 5, 1214 (2006).

[7] G. Hummer and I. G. Kevrekidis, J. Chem. Phys. 118, 10762 (2003).

[8] P. Lepage, e-print arXiv:nucl-th/9706029.
[9] J. Zinn-Justin, Quantum Field Theory and Critical Phenomena (Oxford Science Publications, Oxford, 1993).

[10] H. Kleinert, Path Integrals in Quantum Mechanics, Statistics, Polymer Physics, and Financial Markets (World Scientific, Singapore, 2004).

[11] X.-P. Li and J. G. Broughton, J. Chem. Phys. 86, 5094 (1987)

[12] P. Faccioli, M. Sega, F. Pederiva, and H. Orland, Phys. Rev. Lett. 97, 108101 (2006).

[13] M. Sega, P. Faccioli, F. Pederiva, G. Garberoglio, and H. Orland, Phys. Rev. Lett. 99, 118102 (2007).

[14] E. Autieri, P. Faccioli, M. Sega, F. Pederiva, and H. Orland, J. Chem. Phys. 130, 064106 (2009).

[15] P. Faccioli, J. Phys. Chem. B 112, 13756 (2008).

[16] A. Adib, J. Phys. Chem. B 112, 5910 (2008).

[17] J. W. Negele and H. Orland, Quantum Many-Particle Systems (Perseus Book Publishing, Reading, MA, 1998). 